\title{
Molecular imaging of endothelial activation and mineralization in a mouse model of accelerated atherosclerosis
}

\author{
Guillaume Rucher ${ }^{1}$, Lucie Cameliere ${ }^{1,2}$, Jihene Fendri ${ }^{1,2}$, Antoine Anfray ${ }^{3}$, Ahmed Abbas ${ }^{4}$, Saïd Kamel ${ }^{5,6}$, \\ Quentin Dupas ${ }^{1}$, Nicolas Delcroix ${ }^{7}$, Ludovic Berger ${ }^{1,2}$, Alain Manrique ${ }^{1,8,9^{*}}$ (i) and on behalf of the STOP-AS \\ investigators
}

\begin{abstract}
Purpose: Preclinical imaging of endothelial activation and mineralization using both positron emission tomography (PET) and magnetic resonance (MR) remains scarce.

Procedures: A group of uremic ApoE ${ }^{-/-}(\mathrm{Ur})$, non-uremic $\mathrm{ApoE}^{-/-}(\mathrm{NUr})$, and control C57Bl/6 J mice (Ctl) were investigated. Mineralization process was assessed using sodium fluoride ([18F]NaF) PET, and MR imaging combined with intravenous injection of MPIO-aVCAM-1 was used to evaluate endothelial activation. Micro- and macrocalcifications were evaluated by flame atomic absorption spectroscopy and von Kossa staining, respectively.

Results: Ur mice showed an active and sustained mineralization process compared to Ctl mice $(p=0.002)$ using [18F]NaF PET imaging. Calcium plasma level was increased in Ur $(2.54 \pm 0.09 \mathrm{mM}, n=17)$ compared to NUr and Ctl mice (2.24 $\pm 0.01, n=22$, and $2.14 \pm 0.02, n=27$, respectively; $p<0.0001$ ). Likewise, vascular calcium content was increased in Ur $\left(0.51 \pm 0.06 \mu \mathrm{g} \mathrm{Ca}{ }^{2+}\right.$ per milligram of dry weight aorta, $\left.n=11\right)$ compared to $\operatorname{NUr}(0.27 \pm 0.05, n=9, p=0.013)$ and $\mathrm{Ctl}(0.28 \pm 0.05, n=11, p=0.014)$. Ur mice also had a higher inflammatory state using MPIO-aVCAM-1 MR ( $p$ global $=0.01$, post hoc analysis Ur vs. Ctl $p=0.003$ ) associated with increased VCAM-1 expression ( $p$ global $=0.02$ ). Aortic remodeling at the level of the brachiocephalic trunk, brachiocephalic trunk itself, and aortic arch in Ur mice was also demonstrated using MR.
\end{abstract}

Conclusions: Preclinical molecular imaging allowed in vivo characterization of the early phase of atherosclerosis. [18F]NaF PET showed early and sustained vascular mineralization in uremic $\mathrm{ApoE}^{-/-}$mice. MPIO-aVCAM-1 MR imaging demonstrated aortic endothelial activation, predominantly in segments with vascular remodeling.

Keywords: Positrons emission tomography (PET), Magnetic resonance imaging (MRI), Atherosclerosis, Mineralization, Endothelial activation

\section{Background}

Atherosclerosis is a leading cause of death and disability worldwide and involves several pathophysiological processes including endothelial activation, inflammation, mineralization, and necrosis. Vascular remodeling and the inflammatory state of lesions determine the plaque

\footnotetext{
* Correspondence: manrique@cyceron.fr

${ }^{1}$ Normandie Univ, UNICAEN, EA 4650, GIP Cyceron, 14000 Caen, France

${ }^{8}$ Médecine Nucléaire, CHU de Caen, Avenue de la Côte de Nacre, 14000

Caen, France

Full list of author information is available at the end of the article
}

instability and therefore the risk of subsequent deleterious clinical events (myocardial infarction, stroke). Preliminary clinical studies showed the ability of sodium fluoride $([18 \mathrm{~F}] \mathrm{NaF})$ positron emission tomography (PET) to highlight active mineralization process within the vascular wall $[1,2]$. The deposition of calcium crystals within the plaque during atherosclerosis process is due to phenotypic changes of vascular smooth muscle cells (VSMCs) into osteoblastlike cells [3]. Bone morphogenetic proteins (BMPs), oxydative stress, or changes in pyrophosphate levels are factors that induce osteogenic differentiation in VSMCs. BMP2 is 
recognized as a mediator of both mineralization and local inflammation in pathologic conditions [4] and was found to be expressed in atherosclerotic lesions [5] as an increased expression of alkaline phosphatase [6].

The $\left[{ }^{18} \mathrm{~F}\right]$-fluorodeoxyglucose (FDG) uptake in arterial wall is proportional to macrophage infiltration and reflects the inflammatory state of atherosclerotic lesions [7]. The estimation of soluble biomarkers such as intracellular adhesion molecule-1 (ICAM-1) or vascular cell adhesion molecule-1 (VCAM-1) also underlines the level of endothelial activation. An upregulation of VCAM-1 expression is a feature of inflammatory conditions [8]. Preclinical magnetic resonance (MR) imaging allows the assessment of deep tissues with a non-ionizing imaging method. Associated with an intravenous injection of micron-sized particles of iron oxide (MPIO) conjugated with specific $\alpha \mathrm{VCAM}-1$ antibodies, MR imaging allows the endothelial activation mapping in mice [9].

In a recent review of the literature [10], the Cardiovascular Study Group of the European Society of Molecular Imaging emphasized the lack of preclinical data on the use of [18F]NaF. Particularly, preclinical investigations may help understand the relationship between early microcalcifications demonstrated by $[18 \mathrm{~F}] \mathrm{NaF}$ vascular uptake and both endothelial activation and further vascular remodeling. In the present study, we used both [18F]NaF PET and MPIO$\alpha$ VCAM- 1 MR to assess early endothelial activation and mineralization processes in a mouse model of accelerated atherosclerosis.

\section{Methods}

\section{Animal model}

The institutional animal ethics committee (Comité National de Réflexion Ethique sur l'EXpérimentation Animale CENOMEXA) approved the animal experiments (\#3979), and all experiments were performed with European and French regulation. A control group consisted of 31 control C57Bl/6 J mice (Ctl), a group of non-uremic $25 \mathrm{C} 57 \mathrm{Bl} / 6 \mathrm{~J} \mathrm{ApoE^{-1- }}$ mice (NUr), and a group of uremic $27 \mathrm{C} 57 \mathrm{Bl} / 6 \mathrm{~J} \mathrm{ApoE}^{-/-}$ mice (Ur) were investigated. All animals were male and maintained on a standard chow diet composed by $8.4 \%$ fat, $19.3 \%$ protein, $72.4 \%$ carbohydrates, $0.55 \%$ phosphorus, $0.73 \%$ calcium, $0.16 \%$ magnesium, and $1000 \mathrm{UI} / \mathrm{kg}$ vitamin $\mathrm{D}_{3}$. We induced chronic renal failure by an electrocoagulation of the right kidney at 8 weeks old, followed 2 weeks later by a contralateral nephrectomy, according to a previously described procedure [11]. Analgesia was induced by injection of buprenorphine $\left(0.05 \mathrm{mg} / \mathrm{kg}\right.$, Buprecase ${ }^{\bullet}$, Axience, Pantin, France) administered in preoperative and at $9 \mathrm{~h}$ interval in postoperative during the first $24 \mathrm{~h}$.

\section{In vivo PET acquisitions and reconstruction}

All acquisitions were performed using a dedicated preclinical PET-CT system (Inveon', Siemens Healthcare, Erlangen, Germany). At 12 and 16 weeks old, anesthesia was induced with $5 \%$ isoflurane gas and maintained with isoflurane $2 \%$ gas in a mixed of $\mathrm{O}_{2}$ and $\mathrm{N}_{2} \mathrm{O}$ (1:2). Following attenuation CT acquisition, [18F] NaF injection $(\approx 18 \mathrm{MBq})$ was performed intravenously through a tail vein catheter. Fifty minutes after [18F]NaF injection, a 10-min list mode acquisition of emission data was performed (energy and coincidence windows of $350-650 \mathrm{keV}$ and $3.4 \mathrm{~ns}$, respectively). Image series were reconstructed using 3D-ordered subset expectation maximization (3D-OSEM) with 8 iterations and a zoom factor 2 without scatter correction.

Postprocessing was performed using Carimas v2.4 software (Turku PET Centre, Turku, Finland). The $[18 \mathrm{~F}] \mathrm{NaF}$ activity was represented with a color scale starting just upon the plasma activity determined drawing a volume of interest over the left ventricle with a maximum set to $200 \%$ of the plasma activity measured at the last frame of the data for each examination [12]. An uptake in a region close and above to the heart was considered as a positive aortic $[18 \mathrm{~F}] \mathrm{NaF}$ uptake. Then, aortic uptake was semi-quantitatively determined as the ratio of the SUVmax in a VOI drawn over the visible aortic uptake to the SUVmax of vascular background measured in the inferior vena cava. In addition, global cardiac $[18 \mathrm{~F}] \mathrm{NaF}$ uptake was also measured in a volume of interest encompassing the whole heart normalized to the same vascular background.

\section{MR imaging}

MR experiments were carried out in a second group of animals using a 7-T magnet (Pharmascan ${ }^{\circ}$ Bruker, Billerica, USA) with dual respiratory and ECG gating. Telediastolic T2*-weighted MR images were acquired using a multi-slice sequence: field of view $1809 \times 939 \mathrm{~mm}^{2}$, TR/TE $100 / 4.25$ $\mathrm{ms}, 12$ slices, and voxel $0.1 \times 0.1 \times 0.15 \mathrm{~mm}^{3}$ encompassing the thoracic aorta were performed in 16-week-old mice, with and without the intravenous injection of $200 \mu \mathrm{L}$ of MPIO- $\alpha$ VCAM- 1 .

As previously described [9], microparticles of iron oxide (DynaBeads MyOnes Tosyl Activated, ThermoFisher Scientific, Waltham, USA) were conjugated with antibodies anti- $\alpha$ VCAM-1 (clone A(429), BD BioScience, Franklin Lakes, USA) by incubation at $37^{\circ} \mathrm{C}$ for $48 \mathrm{~h}$. MRI images were analyzed using Osirix v.6.5.2 software. MPIO- $\alpha$ VCAM- 1 binding results in a T2* signal void in the lumen of the vasculature. Intravascular diameters were measured using non-injected MR images on the following segments: (i) the aortic root, (ii) the ascending aorta, (iii) the aorta at the level of the brachiocephalic trunk, (iv) the brachiocephalic trunk, and (v) the aortic arch (Fig. 1). The 


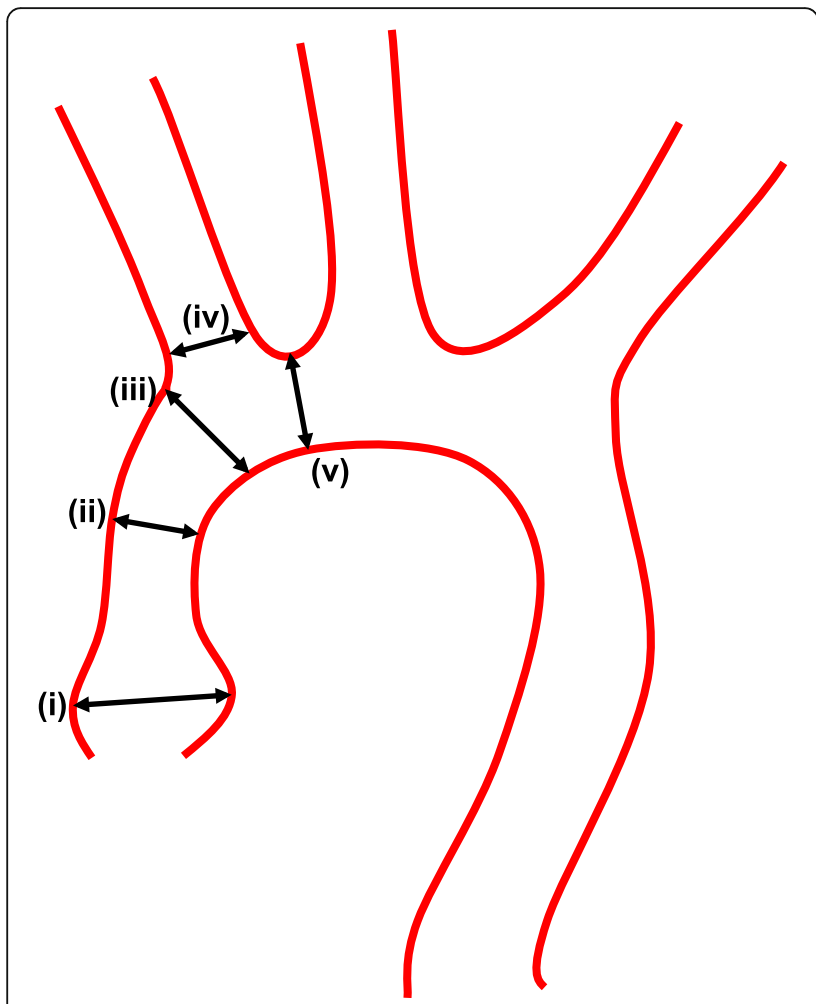

Fig. 1 Scheme of aortic diameters measured in MR images. (i) The aortic root, (ii) the ascending aorta, (iii) the aorta at the level of the brachiocephalic trunk, (iv) the brachiocephalic trunk, and (v) the aortic arch

diameter measurement was indexed to the animal weight measured the day of the MR acquisition.

\section{Tissues samples}

Mice were sacrificed at 16 weeks old. Blood samples were collected to determine the plasma level of urea and calcium using Beckman-Coulter AU5800 autoanalyzer (Miami, USA). Aortas were harvested from the root to the diaphragm. In $11 \mathrm{Ctl}, 9 \mathrm{NUr}$, and 11 Ur, aortic tissue calcium content was measured by flame atomic absorption spectroscopy (FAAS) (Varian AA240, Varian Inc., Palo Alto, CA). After an overnight bath of aortas in a $0.6 \mathrm{~N} \mathrm{HCl}$ solution at $4{ }^{\circ} \mathrm{C}$, FAAS was performed in supernatant. Aortas were dried at $37^{\circ} \mathrm{C}$, then weighted, and the vascular calcium concentration was reported on the dry weight of each aorta.

In $6 \mathrm{Ctl}, 5 \mathrm{NUr}$, and $11 \mathrm{Ur}$, macrocalcifications were assessed using the von Kossa staining. Aortas were dissected under a microscope, perfused with heparinized $(50 \mathrm{U} / \mathrm{mL})$ phosphate-buffered saline 5/100 (PBS), and cryomounted in optimal cutting embedding medium (OCT, Thermo Scientific, Waltham, USA). Ten-micrometer-thick slices were collected each $50 \mu \mathrm{m}$. Cryosections were placed in $5 \%$ silver nitrate solution for
30-60 min then fixed in 5\% sodium-thiosulfate solution for 2-3 min. Sections were digitized using ScanScope CS (Leica Byosystems, Wetzlar, Germany,) and tissue segmentation was manually performed using Aperio ImageScope software v12.3 (Leica Biosystem, Wetzlar, Germany). Data were expressed as the relative proportion of stained tissue to total tissue area.

\section{Western blotting}

Harvested aortas were homogenized in homogenization buffer $(20 \mathrm{mM}$ Tris $\mathrm{HCl}, 150 \mathrm{mM} \mathrm{NaCl}, 1 \mathrm{~m}$ EDTA, $1 \%$ Triton X-100), then sonicated and centrifuged (20,000 G, $\left.15 \mathrm{~min}, 4^{\circ} \mathrm{C}\right)$. Total protein concentrations were determined using the Bradford assay (Bio-Rad, Hercules, USA), and protein was solubilized in Laemmli $4 \times$ buffer $(25 \mathrm{~mL}$ TrisSDS, $20 \mathrm{~mL}$ glycerol, $4 \mathrm{~g}$ SDS, $2 \mathrm{~mL}$ ßmercaptoethanol, $1 \mathrm{mg}$ Bromophenol Blue, $50 \mathrm{~mL} \mathrm{H}_{2} \mathrm{O}$ ). This solution was vortexed, and proteins were denatured using thermal cycler GeneAmp PCR system 2700 (Applied Biosystems, Foster City, USA) at $95^{\circ} \mathrm{C}(3 \mathrm{~min})$. Proteins $(50 \mu \mathrm{g})$ were separated on a 12\% SDS-PAGE gel (86.4 g glycine, $6 \mathrm{~g}$ SDS, $18 \mathrm{~g}$ TrisBase) and transferred to a nitrocellulose membrane (BioRad, Hercules, USA). Blots were blocked with a blocking solution (non-fat dry milk). Primary antibodies were incubated $12 \mathrm{~h}$ at $4{ }^{\circ} \mathrm{C}$ with anti-VCAM- 1 from Cell Signaling (1:1000, VCAM-1 (D2T4N) Rabbit mAb (Mouse Specific), Ozyme distributor, Saint Quentin en Yvelines, France), anti-OPN-R (1:2500, OPN-R Antibody (24H5L3) ABfinity Rabbit mAb, ThermoFisher Scientific, Waltham, USA), and anti-GAPDH (1:1000, GAPDH Loading Control Antibody (MA5-15738), ThermoFisher Scientific, Waltham, USA). After incubation with appropriate horseradish peroxidaseconjugated secondary antibodies for $1.5 \mathrm{~h}$ at room temperature, proteins were visualized by chemiluminescence. Immunoreactive proteins were scanned using Chemidoc XRS (Bio-Rad, Veenendaal, The Netherlands), and intensities were analyzed with ImageJ NIH image processing software (version 1.52a, Bethesda, MD, USA).

\section{Statistical analysis}

Values were expressed as mean \pm SEM. Continuous data were compared using the Wilcoxon signed-rank test or Mann-Whitney $U$ test when appropriate. Multivariate analysis was performed using logistic regression, with post hoc analysis using Tukey's HSD test or odds ratio when appropriate. The KruskalWallis test was used for global comparison between three independent animal groups. For proportions, the chi-square test was used to compare differences between groups. Statistical analyses were performed using JMP 11 (SAS Institute, Cary, NC, USA). A $p$ value $\leq 0.05$ was considered statistically significant. 
a

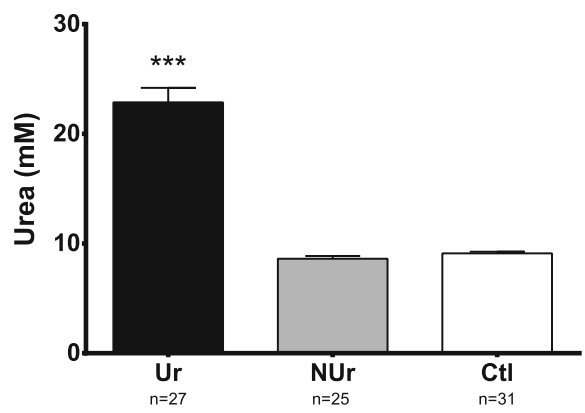

b

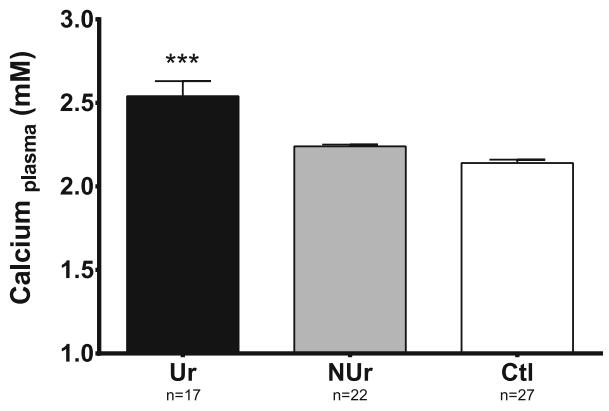

Fig. 2 Urea (a) and calcium (b) plasma level. a Urea plasma level (mM) in uremic ApoE ${ }^{-/-}$mice $(U r, n=27)$, non-uremic ApoE ${ }^{-/-}$mice (NUr, $n=$ 25), and control Bl6 mice (Ctl, $n=31)$. b Calcium plasma level (mM) in uremic ApoE ${ }^{-1-}$ mice $(U r, n=17)$, non-uremic ApoE ${ }^{-/-}$mice $(\mathrm{NUr}, n=22)$, and control mice $(n=27)$. Data are expressed as mean \pm SEM. ${ }^{* * *} p<0.0001$

\section{Results}

\section{Ex vivo assessment}

Urea plasma level was significantly increased in Ur $(22.87 \pm 1.32 \mathrm{mM}, n=27)$ compared to NUr and Ctl mice (respectively $8.62 \pm 0.25 \mathrm{mM}, n=25$, and $9.11 \pm$ $0.15 \mathrm{mM}, n=31 ; p<0.0001$; Fig. 2a). The concentration of calcium in plasma was significantly higher in Ur $(2.54 \pm 0.09 \mathrm{mM}, n=17)$ compared to NUr and Ctl mice (respectively $2.24 \pm 0.01 \mathrm{mM}, n=22$, and $2.14 \pm 0.02 \mathrm{mM}$, $n=27 ; p<0.0001$; Fig. 2b). Flame atomic absorption spectroscopy demonstrated a significant increase of vascular calcium in Ur mice $\left(0.51 \pm 0.06 \mu \mathrm{g}\right.$ of $\mathrm{Ca}^{2+}$ per milligram of dry weight aorta, $n=11)$ compared to NUr

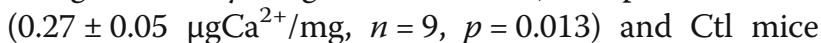
$\left(0.28 \pm 0.05 \mu \mathrm{gCa}^{2+l} \mathrm{mg}, n=11 p=0.014\right.$; Fig. 3$)$. The von
Kossa staining demonstrated a non-significant increase of vascular macrocalcifications in Ur mice $(0.48 \% \pm 0.06$, $n=11$ ) compared to $\mathrm{NUr}$ and Ctl mice (respectively $0.41 \pm 0.03, n=5$, and $0.43 \pm 0.02, n=6$, NS).

\section{PET assessment}

At 12 weeks old, [18F]NaF PET demonstrated an aortic uptake in 8/12 (66\%) Ur mice (Fig. 4), 5/9 (55\%) NUr mice, and $0 / 3(0 \%)$ in Ctl (Table 1$)$. However, only Ur mice $(4 / 9,45 \%)$ had an aortic uptake at 16 weeks old (0/4 in NUr mice and $0 / 4$ in Ctl; Table 1$)$. There was a significant impact of animal group on aortic [18F]NaF uptake $(p=0.01)$, and post hoc analysis showed a significant difference between Ur and Ctl mice $(p=0.002)$.

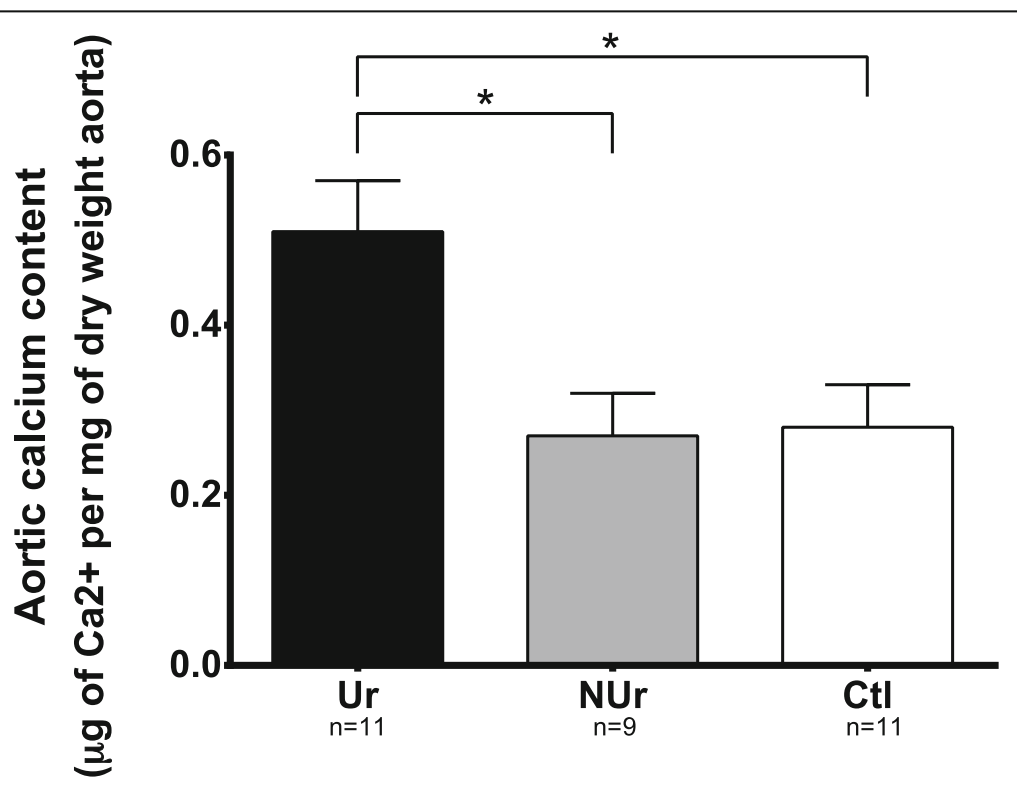

Fig. 3 Aortic tissue calcium content. Values of total aortic tissue calcium content in microgram of Ca ${ }^{2+}$ per milligram of dry weight aorta in uremic ApoE ${ }^{-/-}$mice $(U r, n=11)$, non-uremic ApoE $^{-/-}$mice (NUr, $\left.n=9\right)$, and control Bl6 mice $(C t l, n=11)$. Data are expressed as mean \pm SEM. * $p<0.05$ 


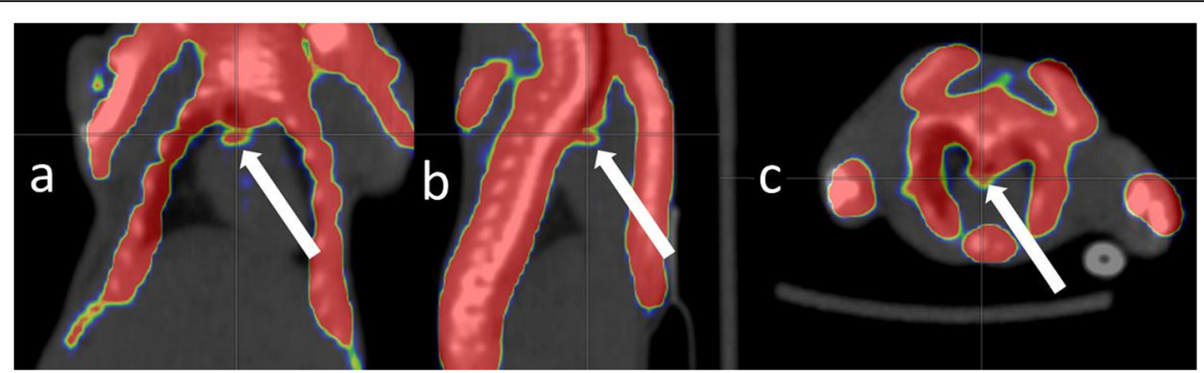

Fig. 4 Aortic [18F]NaF uptake in an uremic $\mathrm{ApoE}^{-/-}$mouse. [18F]NaF PET-CT acquired from 50 to 60 min after the injection of $26 \mathrm{MBq}$ of [18F]NaF in a uremic ApoE ${ }^{-/-}$mouse aged 12 weeks old. a Coronal view. b Sagittal view. c Axial view. White arrow, aortic [18F]NaF uptake

A higher proportion of macrocalcifications on the von Kossa staining was noted in mice without aortic uptake compared to mice with aortic $[18 \mathrm{~F}] \mathrm{NaF}$ uptake at 16 weeks old (PET $-0.89 \% \pm 0.09$ vs. PET $+0.42 \% \pm 0.07$, $p=0.028$ ). There was a global effect of the genotype and the age of the animals on cardiac $[18 \mathrm{~F}] \mathrm{NaF}$ uptake assessment (global $p$ value $<0.05$; Table 2 ). In addition, there was a positive corrrelation between cardiac and aortic [18F]NaF uptake in animals demonstrating positive aortic $[18 \mathrm{~F}] \mathrm{NaF}$ uptake $(r=0.69, p<0.01)$.

\section{MR assessment}

After intravenous MPIO- $\alpha$ VCAM-1 injection, 4/5 (80\%) Ur mice (Fig. 5) and 1/4 (25\%) NUr mice demonstrated a MR signal void in the aortic wall, whereas images were normal in all control mice (Table 3). There was a significant impact of animal group on MPIO- $\alpha$ VCAM-1 binding (global $p$ value $=0.01$ ), and post hoc analysis showed a significant difference between Ur and Ctl mice $(p=0.003)$. Signal void was mostly located within the aortic root. The localizations of MPIO- $\alpha$ VCAM-1 binding is summarized in Table 3.

Morphological MR imaging demonstrated a vascular dilation involving the aorta at the level of the brachiocephalic

Table 1 Contingency table for PET in detecting [18F]NaF aortic uptake $(\mathrm{PET}+)$

\begin{tabular}{llll}
\hline & PET+ & PET- & Total $(n)$ \\
\hline 12 weeks old & & & \\
Ur & $8(66 \%)$ & $4(34 \%)$ & 12 \\
NUr & $5(55 \%)$ & $4(45 \%)$ & 9 \\
Ctl & $0(0 \%)$ & $3(100 \%)$ & 3 \\
16 weeks old & & & \\
Ur & $4(45 \%)$ & $5(55 \%)$ & 9 \\
NUr & $0(0 \%)$ & $4(100 \%)$ & 4 \\
Ctl & $0(0 \%)$ & $4(100 \%)$ & 4 \\
\hline
\end{tabular}

The [18F]NaF activity was represented with a color scale starting just upon the plasma activity determined drawing a volume of interest over the left ventricle with a maximum set to $200 \%$ of the plasma activity measured at the last frame of the data for each examination [12]. An uptake in a region close and above to the heart was considered as a positive aortic [18F]NaF uptake. $\mathrm{Ur}$ uremic $\mathrm{ApoE}^{-/-}, \mathrm{NUr}$ non-uremic $\mathrm{ApoE}^{-/-}, \mathrm{Ct} /$ control mice trunk, the brachiocephalic trunk itself, and the aortic arch in Ur compared to NUr and Ctl mice. The indexed vascular diameters were as follows: aorta at the level of the brachiocephalic trunk, $80.23 \pm 3.50 \mu \mathrm{m} / \mathrm{g}(\mathrm{Ur}, n=4)$ vs. $67.62 \pm$ $3.62(\mathrm{NUr}, n=4)$ and $66.10 \pm 2.94(\mathrm{Ctl}, n=5$, global $p$ value $<0.05)$; brachiocephalic trunk, $36.36 \pm 1.39$ (Ur, $n=4)$ vs. $30.80 \pm 0.65$ (NUr, $n=4$ ) and $31.30 \pm 1.37$ (Ctl, $n=5$, global $p$ value $<0.05)$; aortic arch, $70.49 \pm 2.80(\mathrm{Ur}, n=4)$ vs. $57.99 \pm 2.92$ (NUr, $n=4)$ and $55.70 \pm 1.74$ (Ctl, $n=5$, global $p$ value $<0.05)$; aortic root, $76.23 \pm 7.62 \mu \mathrm{m} / \mathrm{g}(\mathrm{Ur}, n=4)$ vs. $66.69 \pm 3.80(\mathrm{NUr}, n=4)$ and $70.86 \pm 1.51$ (Ctl, $n=5$, NS); and ascending aorta, $66.93 \pm 3.96 \mu \mathrm{m} / \mathrm{g}(\mathrm{Ur}, n=4)$ vs. $60.30 \pm 2.44(\mathrm{NUr}, n=4)$ and $59.36 \pm 3.58(\mathrm{Ctl}, n=5, \mathrm{NS})$.

In addition, we analyzed the results of imaging at 16 weeks (positive vs. negative) with respect to the animal group (Ur, $\mathrm{NUr}, \mathrm{Ctl}$ ) and to the molecular imaging modality (PET and MPIO- $\alpha$ VCAM-1 MRI). The logistic regression demonstrated a significant effect of the animal group on the imaging results $(p<0.001)$, independent of the imaging modality $(p=0.99, \mathrm{~ns})$. The odds ratios for positive imaging results were significant for Ur vs. NUr $(p=0.01)$ and Ur vs. $\mathrm{Ctl}(p<0.001)$ but not for NUr vs. Ctl $(p=0.99, \mathrm{~ns})$.

\section{Western blotting}

The aortic expression of VCAM-1 protein was significantly different between animal groups (Ur $1.27 \pm 0.22$ AU, $n=11$, NUr $0.94 \pm 0.12$, and Ctl $0.56 \pm 0.09$, global $p$ value $=0.02)$, with a significant difference between uremic ApoE $\mathrm{A}^{-/-}$and control mice $(p<0.01$, Fig. 6a). No difference was found between groups for aortic OPN-R protein expression (Fig. 6b).

\section{Discussion}

In this study, a chronic renal failure (CRF) induced an increase of calcium plasma and tissular content in $\mathrm{ApoE}^{-/-}$mice. This study demonstrated the feasibility of [18F]NaF PET to assess in vivo aortic mineralization in a mouse model of accelerated atherosclerosis. In addition, injected MPIO- $\alpha$ VCAM-1 MR imaging allowed the evaluation of active inflammation in this mouse model. 
Table 2 [18F]NaF cardiac and aortic uptake quantification

\begin{tabular}{llll}
\hline & Ur & NUr & $\mathrm{Ctl}$ \\
\hline 12 weeks old & & & \\
Cardiac uptake & $2.2 \pm 0.1$ & $1.9 \pm 0.2$ & $1.8 \pm 0.1$ \\
Aortic uptake & $2.8 \pm 0.3$ & $1.9 \pm 0.3$ & $\mathrm{ND}$ \\
16 weeks old & & & \\
Cardiac uptake & $1.9 \pm 0.2$ & $1.5 \pm 0.1$ & $2 \pm 0.2$ \\
Aortic uptake & $2.6 \pm 0.6$ & $\mathrm{ND}$ & $\mathrm{ND}$
\end{tabular}

[18F]NaF cardiac uptake was assessed in 12 uremic $\mathrm{ApoE}^{-/-}(\mathrm{Ur}), 9$ non-uremic $\mathrm{Apo}^{-1-}$, and 3 control mice at 12 weeks old and in $9 \mathrm{Ur}, 4 \mathrm{NUr}$, and $4 \mathrm{Ctl}$ at 16 weeks old. [18F]NaF aortic uptake was assessed in $8 \mathrm{Ur}$ and $5 \mathrm{NUr}$ at 12 weeks old and in $4 \mathrm{Ur}$ at 16 weeks old. Results are expressed in arbitrary units. ND not done when there was no visible aortic uptake

Our results further demonstrated that the occurrence of mineralization and inflammation processes is associated with vascular remodeling in this animal model.

The CRF is associated with metabolic and endocrine abnormalities, including abnormal calcium and phosphate metabolism and an inflammatory syndrome. In our uremic $\mathrm{ApoE}^{-/-}$mice, the increase of calcium plasma concentration was similar to previous results $[13,14]$. Using a different model, Pulskens et al. also reported a significant increase of calcium plasma level observed 4 weeks after inducing a renal failure using adenine-enriched diet [15]. CRF involves an increased synthesis and secretion of parathyroid hormone (PTH) [13] that is an essential regulator of bone remodeling and calcium homeostasis [16], and results in increased vascular calcification [17]. As previously demonstrated [18], we confirmed using flame atomic absorption spectroscopy an increase of calcium content in aortic tissue of uremic $\mathrm{ApoE}^{-1-}$. On the other hand, the von Kossa staining found no difference between uremic $\mathrm{ApoE}^{-/-}$and other animal groups. These findings are consistent with an early microcalcification process associating an increased calcium vascular content without macroscopic evidence of calcifications. The calcium/phosphate ratio and the vitamin $\mathrm{D}$ in the dietary regimen are important factors to observe macrocalcifications within the aortic wall. Using both a high calcium/phosphate ratio (1.6) and a high vitamin D diet (1540 IU/kg), Massy et al. [13] demonstrated macrocalcifications that we and others did not report when using a similar and lower calcium/phosphate ratio (1.3) and vitamin D intake (600-1000 IU/kg) [14].

Irkle et al. [19] demonstrated that [18F] NaF adsorbs to calcified deposits within plaque with a high affinity and that [18F]NaF PET/CT imaging can distinguish between areas of macro- and microcalcification. However, in vivo preclinical $[18 \mathrm{~F}] \mathrm{NaF}$ PET/CT remains challenging and poorly investigated for the assessment of vascular mineralization process. In the present study, PET data were reconstructed using 3D-OSEM with 8 iterations and a zoom factor 2. Previous results demonstrated that these reconstruction parameters optimized spatial resolution (with a full width at half maximum (FWHM) below $1 \mathrm{~mm}$ ) and allowed the investigation of active aortic mineralization [20] as confirmed by ex vivo measurements of aortic uptake using gamma counter. The present study confirms the feasibility of preclinical $[18 \mathrm{~F}] \mathrm{NaF}$ PET for in vivo assessment of aortic microcalcifications.

$\mathrm{ApoE}^{-/-}$mice develop various lesions of atherosclerosis throughout the arterial system, including foam cell lesions as early as 10 weeks when maintained on chow diet and fibrous plaques after 15 weeks [21]. In addition, uremia further accelerates both atherosclerotic lesions and arterial calcification in ApoE-deficient mice [13]. Our results are consistent with these latter studies. Although it was found in both uremic and non-uremic $\mathrm{ApoE}^{-/-}$mice aged 12 weeks old, an active vascular mineralization process was

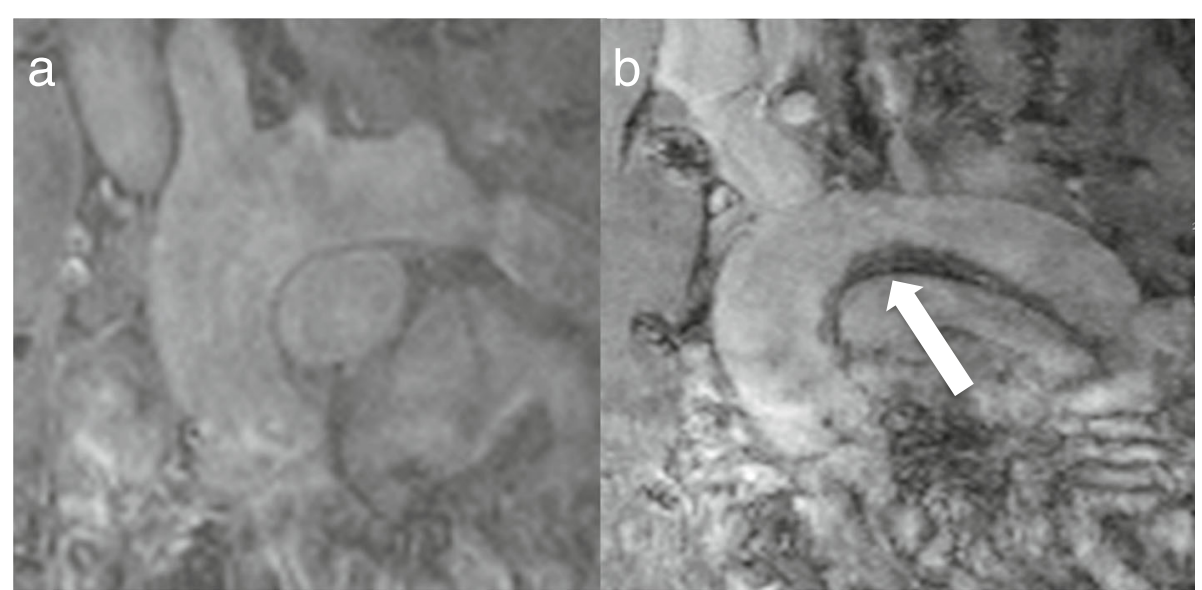

Fig. 5 Aortic arch MPIO-aVCAM-1 binding in an uremic ApoE ${ }^{-1-}$ mice. Telediastolic T2*-weighted MR image acquired before (a) and after (b) the intravenous injection of $200 \mu \mathrm{L}$ microparticles of iron oxide (MPIO) targeted against vascular cell adhesion molecule-1 (VCAM-1). White arrow, MR signal void in aortic arch is due to MPIO-aVCAM-1 binding 
Table 3 Contingency table for MPIO-aVCAM-1 binding localization using $\mathrm{T} 2{ }^{*}$-weighted MR images

\begin{tabular}{llll}
\hline Group of mice & Ur & NUr & $\mathrm{Ctl}$ \\
\hline Aortic root (i) & 4 & 1 & 0 \\
Ascending aorta (ii) & 1 & 1 & 0 \\
Aorta braciocephalic trunk level (iii) & 1 & 0 & 0 \\
Brachiocephalic trunk (iv) & 1 & 0 & 0 \\
Aortic arch (v) & 1 & 0 & 0 \\
Total of animals & $4 / 5$ & $1 / 4$ & $0 / 5$ \\
\hline
\end{tabular}

The site of binding is noted for each animal. An animal may have more than one site of binding. (i) The aortic root, (ii) the ascending aorta, (iii) the aorta at the level of the brachiocephalic trunk, (iv) the brachiocephalic trunk, and (v) the aortic arch. The groups of mice were uremic $\mathrm{ApoE}^{-/-}(\mathrm{Ur}, n=5)$, nonuremic $\mathrm{ApoE}^{-/-}(\mathrm{NUr}, n=5)$, and control mice $(\mathrm{Ctl}, n=5)$

demonstrated only in uremic $\mathrm{ApoE}^{-/-}$mice aged 16 weeks old, suggesting that the CRF induced a sustained process of mineralization. Furthermore, the decrease in $[18 \mathrm{~F}] \mathrm{NaF}$ uptake at 16 weeks old in animals with positive von Kossa staining emphasized that molecular [18F]NaF PET imaging mainly targets the micro- rather than macrocalcifications as previously suggested by ex vivo experiments [19]. Similar results were observed in patients showing positive $[18 \mathrm{~F}] \mathrm{NaF} \mathrm{PET}$ in regions with either no computed tomography $(\mathrm{CT})$ detectable calcification or small foci of spotty calcification whereas macrocalcification remains the main target of CT [22]. Since atherosclerosis is a diffuse process involving the entire arterial system, global $[18 \mathrm{~F}] \mathrm{NaF}$ uptake in the entire heart mesured as evidence for coronary artery atherosclerosis was significantly correlated to aortic uptake and may provide valuable information about the extent of the mineralization process as previously suggested [23].

Active inflammation is an early phenomenon in atherosclerosis [21]. Endothelial activation, a condition associated with most forms of cardiovascular diseases, involves phenotypic changes of the endothelial cell surface that allow leukocyte adherence and diapedesis to injured tissues. Vascular cell adhesion molecule-1 (VCAM-1) is not expressed by quiescent endothelial cells, and the measurement of soluble VCAM-1 is commonly used as a biomarker endothelial activation [8]. Preclinical MR imaging using MPIO targeted against VCAM-1 (MPIO- $\alpha$ VCAM-1) has been shown to demonstrate acute and chronic endothelial activation in various clinically relevant contexts in mice, including chronic renal failure [9]. In the present study, endothelial activation was detectable as a signal void using MPIO$\alpha$ VCAM- 1 T2* MR imaging in $80 \%$ of uremic mice and in $25 \%$ of non-uremic, but was undetectable in control mice. In addition, Western blotting corroborated increased aortic VCAM-1 expression in uremic mice.

Early results previously documented an increased VCAM-1 mRNA expression in aortas from uremic ApoE $^{-1-}$ mice [14]. Nahrendorf et al. [24] developed VCAM-1-targeted nanoparticles that undergo internalization in cells expressing VCAM-1. Using this VINP (VCAM-1 internalizing nanoparticle), they demonstrated the feasibility of non-invasively imaging of VCAM-1 expression in atherosclerosis $\mathrm{ApoE}^{-/-}$mice. They also confirmed early atherosclerotic lesions in juvenile $\mathrm{ApoE}^{-/-}$mice (age, 9 weeks) on a high-cholesterol diet. However, internalized VINP colocalized with VCAM-1 in both endothelial cells and macrophages. Ultrasmall superparamagnetic particles of iron oxide (USPIO) are prone to unspecific extravasation due to their small size and have a lower relaxivity that requires high local concentrations to be detectable $[25,26]$. MPIO- $\alpha$ VCAM-1 combines the larger size of micron-sized particles of iron oxide that prevents unspecific extravasation to a formulation targeted against VCAM-1 that ensures a high sensitivity mapping of endothelial activation. This technique has already been validated in various experimental models of acute or chronic endothelial activation $[9,27]$. In addition to VCAM-1 mapping
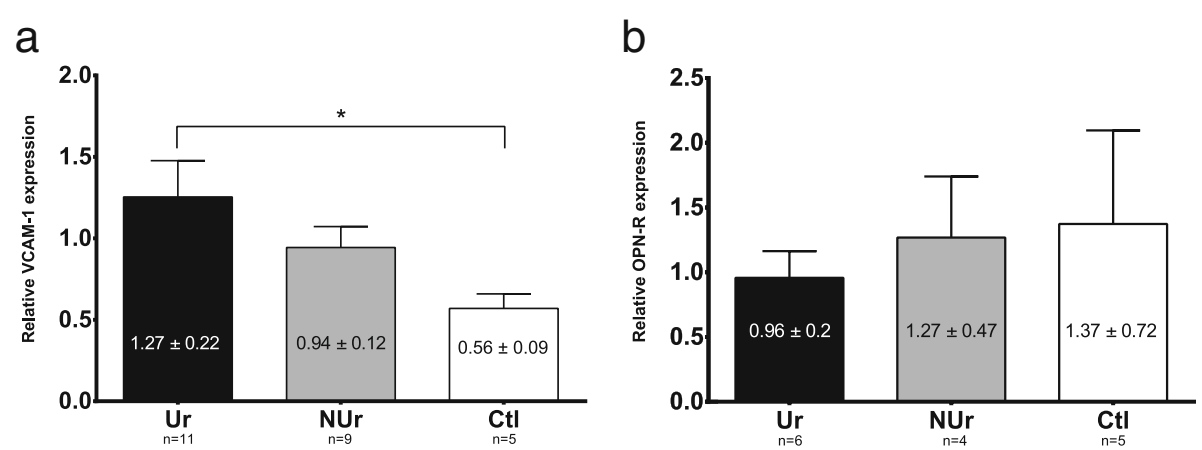

Fig. 6 VCAM-1 (a) and OPN-R (b) aortic protein expression. The protein expression was normalized for each animal by GAPDH protein expression. a There was a significant increase of VCAM-1 protein expression in uremic $\mathrm{ApoE}^{-/-}(\mathrm{Ur}, n=11)$ compared to control Bl6 mice $(\mathrm{Ctl}, n=5$, respectively, $\left.{ }^{*} p<0.05\right)$. b The decrease of OPN-R protein expression in Ur $(n=6)$ compared to NUr and Ctl mice $(n=4$ and $n=5$, respectively) was not significant. Data are expressed as mean \pm SEM 
showing endothelial activation in the aortic arch and the brachiocephalic trunk, we also found a significant dilation of these segments. These enlarged segments match the sites of predilection for atherosclerotic lesion development [21], suggesting an association between endothelial inflammation and structural vascular remodeling.

\section{Conclusions}

In this study, molecular imaging allowed in vivo characterization of the early phase of atherosclerosis. $[18 \mathrm{~F}] \mathrm{NaF}$ PET showed early and sustained vascular mineralization in uremic $\mathrm{ApoE}^{-/-}$mice, a model of accelerated atherosclerosis. In the same model, MPIO- $\alpha$ VCAM-1 MR imaging demonstrated aortic endothelial activation, predominantly in segments with vascular remodeling. In vivo dual assessment of vascular mineralization and endothelial activation opens up new perspectives for future evaluation of the dynamic process of atherosclerosis and new treatment strategies.

\begin{abstract}
Abbreviations
ApoE: Apolipoprotein; BMP: Bone morphogenetic proteins; CRF: Chronic renal failure; Ctl: Control C57BI/6 J; FAAS: Flame atomic absoprtion spectroscopy; FDG: Fluorodeoxyglucose; ICAM: Intracellular adhesion molecular; MPIO: Micron-sized particles of iron oxide; MR: Magnetic resonance; NaF: Sodium fluoride; NUr: Non-uremic; OSEM: Ordered subset expectation maximization; PET: Positron emission tomography; Ur: Uremic; USPIO: Superparamagnetic particles of iron oxide; VCAM: Vascular cell adhesion molecule; VINP: VCAM-1 internalizing nanoparticle; VSMC: Vascular smooth muscle cell
\end{abstract}

\section{Acknowledgements}

The authors want to thank Maelle Guyot and Nicolas Elie for their technical assistance.

\section{Authors' contributions}

GR participated in the design of the study and data acquisition, and drafted the manuscript. AM participated in the design of the study, performed the statistical analysis, and drafted the manuscript. LC, JF, AAn, and AAb participated in the data acquisition and analysis, and revised the manuscript. SK and QD were in charge of the ex vivo experiments and revised the manuscript. ND and LB participated in the design of the study and data analysis, and revised the manuscript. All authors read and approved the final manuscript.

\section{Funding}

This work was conducted as part of the FHU REMOD-VHF project and supported by the French Government, managed by the National Research Agency (ANR) under the program "Investissements d'avenir" with the reference ANR-16-RHUS-0003.

\section{Availability of data and materials}

The datasets used and/or analyzed during the current study are available from the corresponding author on reasonable request.

\section{Ethics approval and consent to participate}

The institutional animal ethics committee approved the animal experiments (\#3979).

\section{Consent for publication}

Not applicable.

\section{Competing interests}

The authors declare that they have no competing interests.

\section{Author details}

${ }^{1}$ Normandie Univ, UNICAEN, EA 4650, GIP Cyceron, 14000 Caen, France. ${ }^{2}$ Chirurgie Vasculaire, CHU de Caen, Avenue de la Côte de Nacre, 14000 Caen, France. ${ }^{3}$ Normandie Univ, UNICAEN, INSERM, UMR-S U1237,

Physiopathology and Imaging of Neurological Disorders (PhIND), 14000 Caen, France. ${ }^{4}$ Normandie Univ, UNICAEN, EPHE, INSERM, U1077, Neuropsychologie et Imagerie de la Mémoire Humaine, 14000 Caen, France. ${ }^{5}$ EA7517, MP3CV, CURS, University of Picardie Jules Verne, Amiens, France. ${ }^{6}$ Biochemistry Laboratory, Amiens University Hospital, Amiens, France. ${ }^{7}$ CNRS, UMS-3048, GIP Cyceron, Campus Jules Horowitz, 14000 Caen, France. ${ }^{8}$ Médecine Nucléaire, CHU de Caen, Avenue de la Côte de Nacre, 14000 Caen, France. ${ }^{9} \mathrm{GIP}$ Cyceron, Campus Jules Horowitz, Boulevard Henri Becquerel, 5229, 14074 Caen, BP, France.

Received: 11 June 2019 Accepted: 9 August 2019

Published online: 22 August 2019

\section{References}

1. Fiz F, Morbelli S, Piccardo A, Bauckneht M, Ferrarazzo G, Pestarino E, et al. 18F-NaF uptake by atherosclerotic plaque on PET/CT imaging: inverse correlation between calcification density and mineral metabolic activity. Journal of Nuclear Medicine. 2015;56:1019-23.

2. Derlin T, Richter U, Bannas P, Begemann P, Buchert R, Mester J, et al. Feasibility of 18F-sodium fluoride PET/CT for imaging of atherosclerotic plaque. Journal of Nuclear Medicine. 2010;51:862-5.

3. Demer LL. A skeleton in the atherosclerosis closet. Circulation. 1995:92:2029-32

4. Johnson RC, Leopold JA, Loscalzo J. Vascular calcification: pathobiological mechanisms and clinical implications. Circulation Research. 2006;99:1044-59.

5. Dhore CR, Cleutjens JPM, Lutgens E, Cleutjens KBJM, Geusens PPM, Kitslaar PJEHM, et al. Differential expression of bone matrix regulatory proteins in human atherosclerotic plaques. Arteriosclerosis, Thrombosis, and Vascular Biology. 2001;21:1998-2003.

6. Aikawa E, Nahrendorf M, Sosnovik D, Lok VM, Jaffer FA, Aikawa M, et al. Multimodality molecular imaging identifies proteolytic and osteogenic activities in early aortic valve disease. Circulation. 2007;115:377-86.

7. Uotila S, Silvola JMU, Saukko P, Nuutila P, Heinonen SE, Ylä-Herttuala S, et al. [18F]Fluorodeoxyglucose uptake in atherosclerotic plaques is associated with reduced coronary flow reserve in mice. Journal of Ultrasound in Medicine. 2014:33:1941-8.

8. Jefferson A, Ruparelia N, Choudhury RP. Exogenous microparticles of iron oxide bind to activated endothelial cells but, unlike monocytes, do not trigger an endothelial response. Theranostics. 2013;3:428-36.

9. Belliere J, Martinez de Lizarrondo S, Choudhury RP, Quenault A, Le Béhot A, Delage $C$, et al. Unmasking silent endothelial activation in the cardiovascular system using molecular magnetic resonance imaging. Theranostics. 2015;5:1187-202.

10. Vigne J, Thackeray J, Essers J, Makowski M, Varasteh Z, Curaj A, et al. Current and emerging preclinical approaches for imaging-based characterization of atherosclerosis. Mol Imaging Biol. 2018;(6):869-87.

11. Gagnon RF, Gallimore B. Characterization of a mouse model of chronic uremia. Urological research. 1988;16:119-26.

12. Joly L, Djaballah W, Koehl G, Mandry D, Dolivet G, Marie P-Y, et al. Aortic inflammation, as assessed by hybrid FDG-PET/CT imaging, is associated with enhanced aortic stiffness in addition to concurrent calcification. Eur J Nucl Med Mol Imaging. 2009;36:979-85.

13. Massy ZA. Uremia accelerates both atherosclerosis and arterial calcification in apolipoprotein E knockout mice. Journal of the American Society of Nephrology. 2004;16:109-16.

14. Bro S, Moeller F, Andersen CB, Olgaard K, Nielsen LB. Increased expression of adhesion molecules in uremic atherosclerosis in apolipoprotein-E-deficient mice. JASN. 2004:15:1495-503.

15. Pulskens WP, Verkaik M, Sheedfar F, van Loon EP, van de Sluis B, Vervloet $M G$, et al. Deregulated renal calcium and phosphate transport during experimental kidney failure. PLoS One [Internet]. 2015 [cited 2018 Jul 22];10. Available from: https://www.ncbi.n/m.nih.gov/pmc/articles/PMC4643984/

16. Swarthout JT, D'Alonzo RC, Selvamurugan N, Partridge NC. Parathyroid hormone-dependent signaling pathways regulating genes in bone cells. Gene. 2002;282:1-17. 
17. Bellasi A, Raggi P. Vascular calcification in chronic kidney disease: usefulness of a marker of vascular damage. J Nephrol. 2011;24(Suppl 18):S11-5.

18. de Oliveira RB, Louvet L, Riser BL, Barreto FC, Benchitrit J, Rezg R, et al. Peritoneal delivery of sodium pyrophosphate blocks the progression of pre-existing vascular calcification in uremic apolipoprotein-E knockout mice. Calcified Tissue International. 2015;97:179-92.

19. Irkle A, Vesey AT, Lewis DY, Skepper JN, Bird JLE, Dweck MR, et al. Identifying active vascular microcalcification by 18F-sodium fluoride positron emission tomography. Nature Communications. 2015;6:7495.

20. Rucher G, Cameliere L, Fendri J, Abbas A, Dupont K, Kamel S, et al. Performance evaluation of a dedicated preclinical PET/CT system for the assessment of mineralization process in a mouse model of atherosclerosis. Mol Imaging Biol. 2018.

21. Nakashima Y, Plump AS, Raines EW, Breslow JL, Ross R. ApoE-deficient mice develop lesions of all phases of atherosclerosis throughout the arterial tree. Arteriosclerosis, thrombosis, and vascular biology. 1994;14:133-40.

22. Blomberg BA, de Jong PA, Thomassen A, Lam MGE, Vach W, Olsen MH, et al. Thoracic aorta calcification but not inflammation is associated with increased cardiovascular disease risk: results of the CAMONA study. Eur J Nucl Med Mol Imaging. 2017;44:249-58.

23. Mohsen B, Babak S, Mehta NN, Torigian DA, Werner T, Mohler E, et al. Detection and global quantification of cardiovascular molecular calcification by fluoro18-fluoride positron emission tomography/computed tomography--a novel concept. Hell J Nucl Med. 2011;14:114-20.

24. Nahrendorf M, Jaffer FA, Kelly KA, Sosnovik DE, Aikawa E, Libby P, et al. Noninvasive vascular cell adhesion molecule-1 imaging identifies inflammatory activation of cells in atherosclerosis. Circulation. 2006;114:1504-11.

25. Gauberti M, Montagne A, Quenault A, Vivien D. Molecular magnetic resonance imaging of brain-immune interactions. Front Cell Neurosci. 2014;8:389-9.

26. Thorek DL, Tsourkas A. Size, charge and concentration dependent uptake of iron oxide particles by non-phagocytic cells. Biomaterials. 2008;29:3583-90.

27. Gauberti M, Montagne A, Marcos-Contreras OA, Le Béhot A, Maubert E, Vivien D. Ultra-sensitive molecular MRI of vascular cell adhesion molecule-1 reveals a dynamic inflammatory penumbra after strokes. Stroke. 2013;44:1988-96.

\section{Publisher's Note}

Springer Nature remains neutral with regard to jurisdictional claims in published maps and institutional affiliations.

\section{Submit your manuscript to a SpringerOpen ${ }^{\circ}$ journal and benefit from:}

- Convenient online submission

- Rigorous peer review

- Open access: articles freely available online

High visibility within the field

- Retaining the copyright to your article

Submit your next manuscript at $\boldsymbol{\nabla}$ springeropen.com 